\title{
Object Oriented Sparse Linear Solver Component for Power System Analysis
}

\author{
Hazlie Mokhlis, and Khalid Mohamed Nor, Senior Member, IEEE
}

\begin{abstract}
Thls paper describes the development of a sparse Inear solver component for solving linear equation in poiver system analysis. The solver is developed into a component by using Object Oriented Programming and Component Based Development methodologies. This component is then integrated with the load flow and fault analysis components as power system analysis software. By developing the solver and the power system analyses into a different component, the engineering analysis becomes independent from the sparse linear solver. Therefore, the solver can be replaced with other solver, which may be proprietary code, or when better or improved solver becomes available in future. The replacement will not cause any need to modify the load flow and fault analysis 'components. By using Component Based Development, the software becomes flexible to be updated and extended.
\end{abstract}

Keywords--LInear solver, component, object-oriented power system - model, component-based system development. Nomenclature

\section{INTRODUCTION}

$T^{n}$ any type of power system analyses, a sparse linear solver to solve matrix equation such as inversion is the core part of such analysis. Without this solver, power systen analyses cannot be solved. The solver will determine the accuracy of the solution and also the analysis solution time, whether slow or fast. Thus, it is very important to have a reliable solver, which can solve matrix equation involving various types of large scale sparse matrices in power system analysis. The solution time taken in solving matrix equation also needs to be fast especially for real time application.

For along time, it has been a practice in power system analysis software development to combine the electrical analysis together with the matrix solver such as in load flow application [3, 4]. This approach made the electrical analysis and the solver analysis in the program being coupled together. Modification on particular variable or function may propagate to the whole source codes of both parts, which lead to a massive modification. Due to this problem, software maintenance became difficult, time consuming and cosily. This problem can be avoided by developing the solver separately from the engineering analysis before combining,them together. In order to develop power system analysis software from different part, an Object Oriented Progtamming (OOP) and Component based development (CBD) methodologies can be applied.

This work was supported in part by the University of Malaya, Kuala Lumpur Malaysia, under JRPA grant project.

Hazlie Mokhlis is with the Department of Elcctrical Engineering. University of Malaya, (e-mail: laazli(ü)um.ed(1.my)

Khalid Mohamed Nor is with the Department of Electrical Finginecring. University of Mataya (e-mail: khalid@um.edu.ny).
By using OOP, the problem of upgrading can be reduced. There are many reports on the application of OOP for power system application [5-7]. Although OOP has shown to help in maintaining soliware, this is only at the source codes level since the codes still have to be recompiled whenever their functionalities need to be extended. These limitations can be solved by using CBD, where application is built in whole or in part from existing pieces [8]. Component provides many advantageous to software development such as it allow cross language, simpler to understand and use, and support visual programming. These advantageous have lead to its usage in developing power system analysis such as in [7], where OOP and CBD were both applied. By using CBD, the software became reusable since each of components inside the application is independent between each other. Changing on any component will not affect the rests. The functionality of the component can also be extended without recompiling it.

Since the CBD methodology provides a way of developing software from various components, power system analysis and sparse lincar solver can be developed into different components. The engineering analysis will be able to use the this solver component to solve linear equation involve in it. However, 10 build from scratch the solver is time consuming since it involves complex mathematical analysis, which may not be easy to understand and develop in a short time. Therefore, it is an advantage to use an existing solver (source codes) that available in the market or public domain rather than developing it from scratch. This solver can be prepared into a component before integrated it with power system analysis component to be power system analysis soltware.

There are few examples of public domain solvers such as 'Spooles', 'SuperLU' and 'Boeing' $[1,2,3\}$. These solvers have been tested and proven to work in solving various type of sparse matrix. By using such existing solver, cost and time of power system software development can be reduced. Furthermore, the electrical analysis will be belter design since the developer can concentrate in the engineering part and leave the detail of mathematic to the mathematicians.

In this project, 'SuperLU' is used as the solver and has been developed into a component. Although it is a putblic domain code, it still has the same capability comparey to other in solving sparse linear equation. It uses many latest techniques, such as graph reduction technique in matrix factorization. Furthermore, it can also solve very unsynmetrical matrices. The package comes with real and complex matrices solver, in both single and double precision versions.

This paper will present a report on the development of an object oriented natrix solver component. Its application in toad flow and fault analyses application will also be discussed. 


\section{INTRODUCTION TO SUPERLU}

SuperLU is a general purpose library for the direct solution of large, sparse, non-symmetric systems of linear equations on high performance machines. It was developed by the University of California, through Lawrence Berkeley National Laboratory. It is a license free, which can be used for research with particular conditions. Tlie library is written in ANSI $\mathrm{C}$ and is callable from either $\mathrm{C}$ or FORTRAN. Since it is implemented in ANSI $\mathrm{C}$, it need to be compiled with standard ANSI $C$ compilers.

SuperLU contains a set of subroutines libraries for solving sparse linear system of

$$
\mathbf{A} \cdot \mathbf{X}=\mathbf{B}
$$

where $\mathbf{A}$ is a square, nonsingular, $n \times n$ sparse matrix and $\mathbf{X}$ and $\mathbf{B}$ are dense $n \times$ nrhs matrices, where $n r h s$ is the number of right-hand sides and also the solution vectors. SuperLU provides functionality to solve equation 1 for both real and complex matrices, in both single and double precision.

Basically, SuperLU uses LU decomposition technique in solving linear equation. The library routines perform LU decomposition with partial pivoting. Matrix $\mathbf{A}$ is factorized to decompose into $\mathrm{L}$ and $\mathrm{U}$ matrices as follow,

$$
\mathbf{A} \cdot \mathbf{X}=(\mathbf{L} \cdot \mathbf{U}) \cdot \mathbf{X}=\mathbf{B}
$$

by first solving for vector $\mathbf{y}$ such that

L. $\mathbf{y}=\mathbf{B}$

and then solving

$$
\mathbf{U} \cdot \mathbf{X}=\mathbf{y}
$$

to get the solution of $X$. Equation 3 and 4 are solved using forward and back substitution. The LU factorization routines can handle non-square matrices of $\mathbf{A}$ but the solution for equation 3 and 4 are performed only for square matrices. Matrix $\mathbf{A}$ can be in a symnetrical or very unsymmetrical structure.

SuperLU also provides routines to improve backward stability, equilibrate the system, estimate the condition number, calculate the relative backward error, and estimate error bounds for the refined solutions.

\section{II!. CLASSES FOR SPARSE LINEAR SOLVER}

In this project, the SuperLU source codes are rewritten into OOP since the original codes are based on ANSI $\mathrm{C}$ with a structural programming. There are also some modification in the codes such as changing the maximum number of precision that allowed for a double data type. This is because the library was built under UNIX system, which is different from a PC (Personal computer).

The source codes in the SuperLU package are grouped into particular classes. This grouping is based on the data type of the matrices, whether conplex number or real number and also the type of accuracy required for the analysis, whether single or double precision. The classes and it relationship is shown in Figure 1.

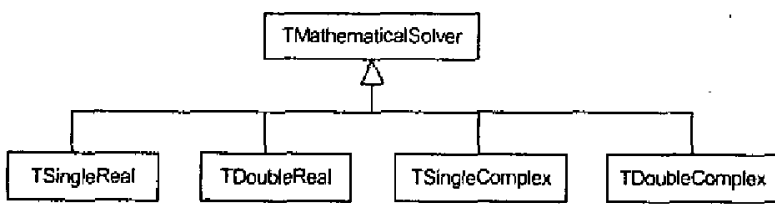

Fig I Sparse Linear Solver Class Diagram
Four types of classes that are TSingleReal, TDoubleReal, TSingleComplex, and TDoubleComplex are derived from the base class, which is the TMathemathicalSolver class. This relationship between the base class and derived classes is called inheritance. The base class contains common attributes of data and methods (functions) for the derived classes such as the type of number for data, whether real or complex number. Therefore, the same methods that required in the derived classes do not need to be rewritten again in those classes.

The derived classes are defined with polymorphism relationship with the base class. By doing so, user can access a same method with different operation depending on the defined object, whether single or double precision. Besides the existing functions in the solver, other basic operations such as multiplication and addition involve one-dimensional sparse matrix were also added into this solver classes. As an example fragment of $\mathrm{C}++$ code (header file) for single real solver. is presented as follows,

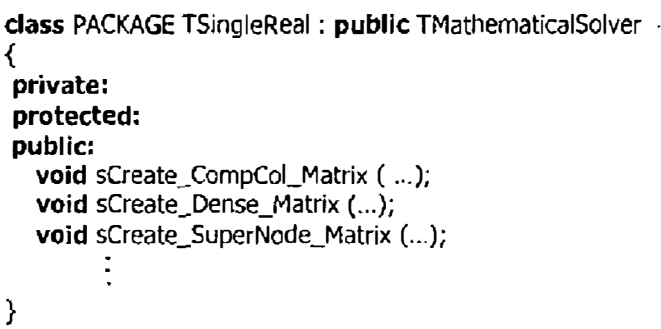

The above header file shows some examples of the methods (functions) inside the Single Real class. These methods have it own task in solving the equation. The method of sCreate_CompCol Matrix and sCreate_Dense_Matrix for instance are called to set up matrices $\mathbf{A}$ and $\mathbf{B}$, respectively, in the data structure internally used by SuperLU. Since the Single Real class is derived from the base class of the Mathematical Solver class, all the data and methods, which was defined as a public or protected type.in the base class can be accessed directly by it. This also applied for other derived classes i.e Double Real, Single Complex and Double Complex. By using classes, the solver becomes reusable that can be extended with other functionility.

\section{COMPONENT DEVELOPMENT AND INTEGRATION}

The discussed mathematical solver classes are packed into a package. In $\mathrm{C}++$ Builder, a package is a special dynamic link library used by $\mathrm{C}++$ application and the Integrated Development Environment (IDE), or both. This package consists of files with the extension type of $\mathrm{BPL}, \mathrm{BPI}, \mathrm{OBJ}$, and LIB. This package is then installed so as it will be registered in the IDE component palette. The same procedures were applied in developing load flow and fault analysis components. The component palettes are now ready to be used.

In order to deliver components to user, both 'BPl' and 'BPL' files as well header files with the extension ' $h$ ' need to be supplied. These files are sufficient for application that use component in runtime application. However, applications that are linked statically to a component, additional files with extension of 'LIB' and 'OBJ' type are also need to be supplied. The supplied header files contain only public members with their protected and private 
members are taken out. The reason is to hide members that are not accessible in the derived classes from misused.

In order to integrate the components, all the required components need to be installed first. Then, a main program application is created and all the required functions of the components are called in the main program. Besides the usual application under DOS environment, the load flow and fault component also have been successfully integrated with Graphical User Interface (GUI) components and other component as user-friendly Visual Power System Analysis Tool software [8]. This tool is built by architecting component systens that play the main role in drawing oneline diagram, capturing database, and performing analysis such as load now, symmetrical and unsymmetrical faults. The interactions between these compenents in the developed software are shown in Figure 2.

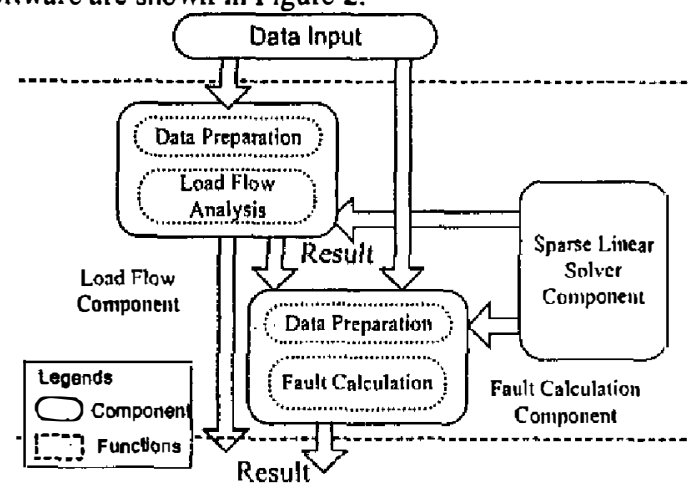

Fig 2 Interactions between Components

In the above figure, the load flow and fault components use sparse linear solver component. The functions of the solver can used to solve equation by calling them in the load flow and fault components. In order to do this, the solver is defined in both component. For fault component, it can receives analysis result from the load flow component such as the voltage on buses, or it can also analyze data directly from the data input.

The data for analysis are supplied by user through the data input component. This component could be a database or a group of functions to read text files. In our data input component, user has the choice to provide data by reading text files in the format of IEEE or from database. The supplied data will be processed first in the Data preparation function inside the load flow and fault component. These data will be prepared according to the data structure uses in load flow and fault analysis. By having these function, load flow and fault analyses components are independent from the structure of the data supplied to it. Thus, any data with different data structure can be supplied as long as it is the right data. The functions can also be considered as an interfacentayer for other application or components to communicate with the load flow and fault components.

The interaction between components shows independency between components. Modification in any of the analysis components or the solver component inside this application will not affect each other. The solver component can also be replaced with other suitable one without affecting the load flow and fault analysis components. Therefore, at any time a better solver could be chosen to replace the existing component in the application.
By developing application based on components, adding, changing or modifying any component in the application is possible without affecting other components. Because of this, other data input component based on other data format likes PSSE or other types of analysis such as stability analysis components can also be integrated into this application. Another important advantage of using component is that it can be added with other derived classes without need to recompile the existing compontent. For an example, if we want to create a new function such as 10 get an inversion matrix, what we need to do is to creale a new componcint class by deriving it fiom llic base class. Only this component is compiled and not the base class.

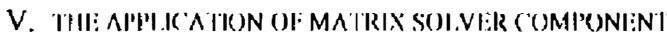

In order to use Superl.U solver, the involve matrices in load flow and fault analyses need to be prepared according to the matrix storage fornat use in SuperLU. The storage format is called compressed column format. Basically, the matrix $A$ in the equation 1 needs to be stored in three different one dimensional arrays. The arrays are referred to as $a[], a s u b[]$ and $x a[]$, which stores the nonzero coefficients of matrix $A$, their row indices, and the indices indicating the beginning of each column in the coefficient and row index arrays. The total number of the nonzero element of the matrix $\mathbf{A}$ also needs to be specified.

\section{A. Fault Analysis Application}

In fault analysis, matrix inversion is required to get a bus impedance matrix. This matrix is obtained by inversing bus admittance maltix of the power system network. The diagonal elements of the bus impedance matrix, which are the Thevenin impedances of the network is used to calculate the fault current at various buses.

Unfortunately, the SuperLU package does not provide an inversion operation since it uses the LU decomposition technique in solving equation 1, which not involving any inversion process. However, we can manipulate the functions provided in SuperLU to develop a function for inversion purpose. Using the LU decomposition provided by SuperLU and back substitution routines, it is possible to find the inverse of a matrix column by column. To explain this, consider a linear set of system of equation 1 with matrix $\mathbf{A}$ has dimensional of $\boldsymbol{n} \times \boldsymbol{n}$. The developed inversion function will factorize matrix $\mathbf{A}$ once to develop $\mathbf{L}$ and $\mathbf{U}$ matrices. Matrix B is supplied with a value of 1 in the first row and the rest of the elements are zero. These matrices then are supplied into a SuperLU solving routine to get the $\mathrm{X}$ matrix of the equation. The result of this $\mathbf{X}$ matrix is the elements of the first column of the inverse of matrix $A$. The same process is repeated by changing the value one of matrix $\mathbf{B}$ into the second row to get the second column of the inverse matrix $A$. These processes are continued until all the columns of the inverse matrix $\mathbf{A}$ are obtained.

\section{B. Load Flow Application}

Different from fault analysis application, load flow application would be able to tise directly the routines provided in SuperLU to solve the involve equation. For Newton Raplison (NR) load llow analysis, the involve equation is [9]: 


$$
\left[\begin{array}{ll}
H & N \\
J & L
\end{array}\right]\left[\begin{array}{l}
\Delta \theta \\
\Delta V / V
\end{array}\right]=\left[\begin{array}{l}
\Delta P \\
\Delta Q
\end{array}\right]
$$

whereas for Fast Decoupled (FD) method, it has the same form of equation as in NR but with two decoupled equations [10]:

$[\Delta P / V]=\left[B^{\prime}\right][\Delta \theta] \quad$ (7) $\quad[\Delta Q / V]=\left[B^{\prime \prime}\right][\Delta V]$

Where,

$\Delta^{\rho}, \Delta Q$ : active and reactive power mismatch vectors

$\Delta V, \Delta \theta:$ voltage magnitude and angle correction vectors

$B_{i k}^{\prime}=1 / X_{i k} \quad-\quad B_{i k}^{\prime \prime}=X_{i k} /\left(R_{i k}^{2}+X_{i k}^{2}\right)$

$B_{i j}^{\prime}=-\sum_{i \in k} 1 / X_{i k} \quad B_{i i}^{\prime \prime}=-\sum_{i \in k} B_{i k}^{\prime \prime}+S_{i k} / 2$

( $R$ and $X$ are the resistance and reactance of transmission line)

In order to solve equation 6,7 and 8 of the NR and FD methods using the sparse linear solver solver component, the Jacobian matrix of NR and FD methods need to be prepared first according to the SuperLU format. A function called Construct structure is developed in both component to prepare the $a s u b[]$ and $x a[]$ arrays. Functions to develop one dimensional arrays for Jacobian!], $\boldsymbol{B}^{\prime}[]$ and $\boldsymbol{B}^{\prime}$ '[] are also developed in the load flow component.

The process of developing the data structure according to SuperLU format for NR method is shown in the flow chart of Figure 3. The flow chart shows how data structure for $\mathbf{H}$ and $\mathbf{J}$ matrices of the Jacobian matrix are determined. The process is starts by setting three variables to their initial value. These variables are col, which represented the column number of matrices $\mathbf{H}$ and $\mathbf{J}$, the index indicates the index number of $a s u b$, and the nonzero variable indicates for the total number of non-zero elements in the Jacobian matrix. Then, the connections between two busses (send bus and receive bus) are checked whether they are equal to the col value or not. If one of them is equal to the col, the receive bus is then checked whether equal to col or not, and then the asub is taken value accordingly as shown in the flow chart and the nonzero value is increased by one. This process is repeated until all the branch data have been checked ( $i$ is equal to the total number of branch). After this, a sorting process is done to rearrange the asub array according to the order of row number of the matrix. This is because the connections data between two busses usually not in appropriate order.

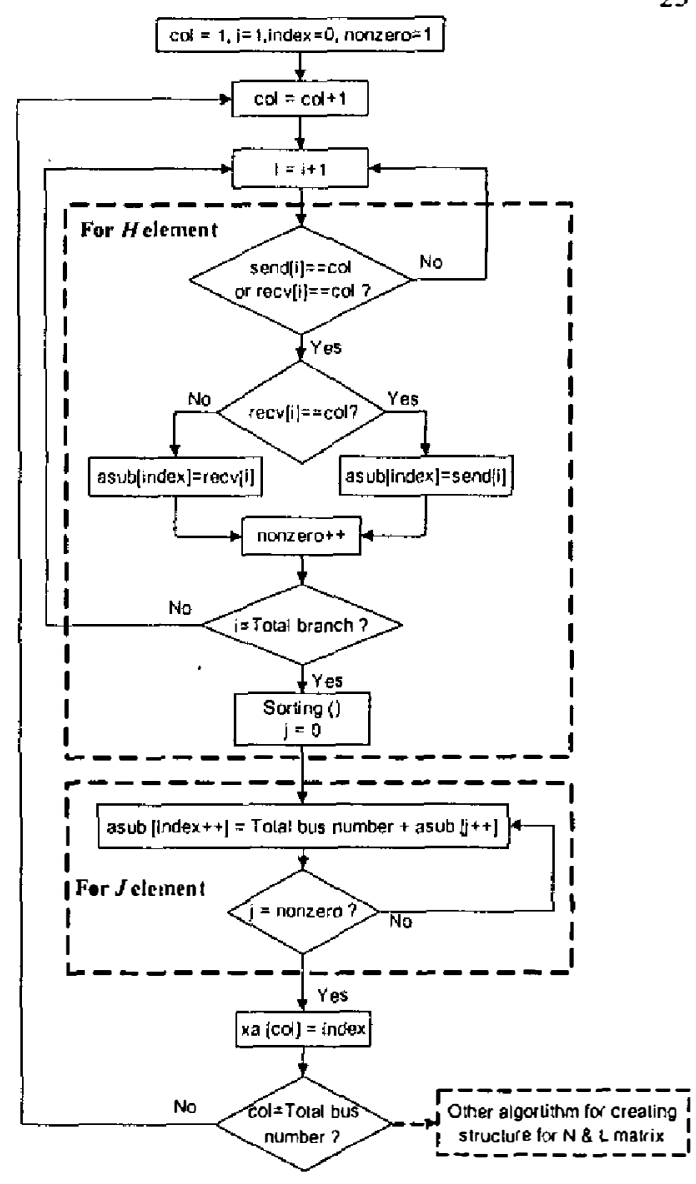

Fig 3 Data structure development in NR method

The next process is to develop the structure of the matrix $\mathbf{J}$. For this the $\boldsymbol{a s u b}$ of the $\mathbf{H}$ matrix, which has been found are used. After all the $\boldsymbol{a s u b}$ of the nonzero element of the $\mathbf{J}$ matrix have been found, the $x \boldsymbol{a}$ indicates the total number of non zero values in one column of the Jacobian matrix is taken the index value. The process is end when all the col value is equal to the total bus number of the system.

For the $\mathbf{N}$ and $\mathbf{L}$ matrices, the data structure development is by manipulating all the result obtained from $\mathbf{H}$ and $\mathbf{J}$ matrices. Similar process is also taken in developing bus admittance matrix in fault analysis to fulfill the storage format of SuperLU.

\section{COMPONENT PERFORMANCE EVALUATION}

The objective of this test is to evaluate the performance of the software that developed based on the component over the non component software in term of execution time. For this purpose, two software applications are deveslsped. using $\mathrm{C}++$ Borland Builder version 6.0 for solving load flow analysis. The first one is integrated from the discussed components and another one is developed based on OOP only. The execution times are taken for solving load flow analysis using both methods and matrix factorization operation involve in the NR method. The test is carried out by using Pentium III, $450 \mathrm{Mhz}$, RAM 64 MB (Personal Computer). The data tests are from IEEE test data involving 118 and 300 bus system and TNB data of 664 bus systems. 
Table 2 Newton Raphşon Load Flow Execution Time

\begin{tabular}{|l|c|c|}
\hline \multirow{2}{*}{ System } & \multicolumn{2}{|c|}{ CPU time (in seconds) } \\
\cline { 2 - 3 } & Componen! & Non Component \\
\hline I18 Bus & 0.16 & 0.10 \\
\hline 300 Bus & 1.93 & 1.36 \\
\hline 664 Bus & 11.81 & 10.49 \\
\hline
\end{tabular}

I'able 3 Fast Decoupled Execulion lime

\begin{tabular}{|l|c|c|}
\hline Systen & \multicolumn{2}{|c|}{ CP[] (inic (In seconts) } \\
\cline { 2 - 3 } & Componenl & Non (omponenl \\
\hline 118 Bus & 0.019 & 0.012 \\
\hline 300 Bus & 0.058 & 0.039 \\
\hline 604 Bus & 0.129 & 0.082 \\
\hline
\end{tabular}

Table 4 Mutrix liacfurizations and laversiun Lixceutient lime

\begin{tabular}{|l|c|c|}
\hline \multirow{2}{*}{ Matrix Size } & \multicolumn{3}{|c|}{ CPU dime(in seconds) } \\
\cline { 2 - 3 } & Component & Non omponent \\
\hline $236 \times 236$ & 0.05 & 0.04 \\
\hline $600 \times 600$ & 0.64 & 0.45 \\
\hline $1328 \times 1328$ & 3.89 & 3.18 \\
\hline
\end{tabular}

All the load flow analysis tests show convergence creteria, which prove the ability of SuperLU in solving matrix equation involving large scale sparse matrices. The execution time of solution is presented in table 2,3 and 4 . Its clear that execution time of application based on component requires more time compared to the non-component. The extra execution time is caused by the overhead given by object oriented classes, where a lot of passing massages are involved. As the number of busses increase, the difference in execution time became smaller. Moreover if the test is run under a more powerful processor, the execution time can be reduced.

\section{CONCLUSION}

This paper has presented the application of public donain source code, which was developed into a sparse linear solver component. The integration of the component with load tlow and fault components has been explained. Although the performance test shows an extra execution time, it not so significant compared with the benefits offered by CBD. Writing more efficient codes or using more powerful computer can reduce the extra time. Since computer processor speed continues to increase, execution time will not be a major handicap in using CBD approach.

By developing the power system software based on component, maintenance is easier as any change in any component does not propagate and affect other components. This development also shows the potential of CBD in developing power system analysis components as off-theshelf products. Power system software developer can therefore maximize time and energy in developing high quality components of his expertise, letting other experts, the non-power system components developers, develop and extend functionalities of other components. This will minimize the cost of developing and maintaining high quality software.

\section{ACKNOWLEUGMENT}

The authors gratefully acknowledge the assistance rendered by the Department of Electrical Engineering and the Faculty of Engineering, University of Malaya in the work reported in this paper.

\section{RHEFERENCES}

[1] "SPOOI.ES 2.2: Sparse Object Oriented Linear Equations Solver", which is availathlc al

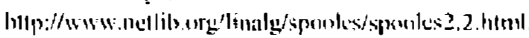

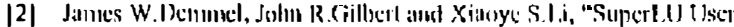
(itidle", which is available at

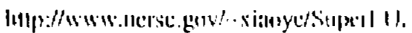

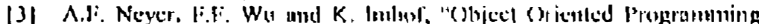

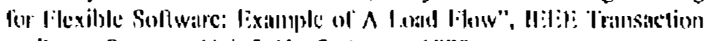
on lower Syslcurs, Vol. 5, No. 3, August 1900.

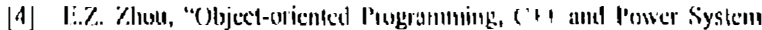
Simulation", IEEE Transaction on Power Systems, Vol.11. No. 1. libruasy $19 \%$.

[5] B, Hakavik, A.T.llolen, "Power System Modeling and Sparse Matrix Opcrations Using Object-Oriented l'roglatmming", Il:til: Trinn. (On Power System, Vol. 9, No. 2, May. 1994.

[6] Joley M., Bose A., Mitchell W. and liaustini $\Lambda .:$ "An (Jbjecl Based Graphical User Interface for Power Systens", IEl:E Transactions on Power Systems, Vol. 8, No. I, liebruary 1993

(7) Khalid M. Nor, Taufiq $\wedge$. Gani, Hazlic Mokhlis, "The Applicalion of Component l3ased Mellodology in I)eveloping Visual P'ower System Analysis Tool", Proceeding of the $22^{\text {nd }}$ conference on IEEII PES PICA, Sydiney, 2000.

(8] Chappel, David, "The Next Wave: Component Soltware Enters the Mainstream", White Papers, which is available al listp://www.rational.com.

[9] W.F Tinney and C.E Hart,"Power flow solution by Newton's method", IEEE Trans, (Power App. Sys), vol. PAS-86, pp.1449-1456, Nov. 1967.

[10] B. Siott and O. Asac, "Fast Decoupled Load Flow ", IEl:E Trans. (Powet App. Sys), vol. PAS-93, pp.859-869, May/June. 1974. 